\title{
A Low Cost Implementation of an Existing Hands-on Laboratory Experiment in Electronic Engineering
}

\author{
http://dx.doi.org/10.3991/ijep.v4i4.3707 \\ C. Onime ${ }^{1}$, J. Uhomoibhi ${ }^{2}$ and M. Zennaro ${ }^{1}$ \\ ${ }^{1}$ Abdus Salam International Centre for Theoretical Physics (ICTP), Trieste, Italy \\ ${ }^{2}$ University of Ulster, Belfast, Northern Ireland
}

\begin{abstract}
In engineering the pedagogical content of most formative programs includes a significant amount of practical laboratory hands-on activity designed to deliver knowledge acquisition from actual experience alongside traditional face-to-face classroom based lectures and tutorials; this hands-on aspect is not always adequately addressed by current e-learning platforms.
\end{abstract}

An innovative approach to e-learning in engineering, named computer aided engineering education (CAEE) is about the use of computer aids for the enhanced, interactive delivery of educational materials in different fields of engineering through two separate but related components; one for classroom and another for practical hands-on laboratory work. The component for hands-on laboratory practical work focuses on the use of mixed reality (video-based augmented reality) tools on mobile devices/platforms.

This paper presents the computer aided engineering education (CAEE) implementation of a laboratory experiment in micro-electronics that highlights some features such as the ability to closely implement an existing laboratory based hands-on experiment with lower associated costs and the ability to conduct the experiment off-line while maintaining existing pedagogical contents and standards.

Index Terms-Augmented reality, computer aid, laboratory experiment, tutorial exercise.

\section{INTRODUCTION}

In classical electronic engineering hands-on practical laboratories, bread-boards are often used as the basis for most basic modular circuit based experiments. Breadboards allow the interconnection/arrangement/wiring of low level (basic) components such as resistors, capacitors and diodes into functional circuits. With the advent of micro-processors and other micro-electronic components, the bread-board is used to interconnect/wire both lowlevel components and high level modular devices such as large scale (LSI) and very large scale integrated (VLSI) circuits such as micro-processors together.

In many experiments, especially those that involve the use of high level modular devices the intended learning outcome is not primarily about the interconnection of circuitry, but is sometimes about programming/obtaining a specific functionality from the high level device and familiarization with the high level device. For these types of experiments, pre-fabricated boards (containing some components already embedded in the circuit board) play the same role as the bread-boards and allows the interconnection of highly modularized (integrated) components such as sensors and other processing devices together, while minimizing interconnection errors of expensive components.

The use of pre-fabricated boards also allows the learner to focus on the high level objectives rather than the lowlevel circuitry as some of the components are already wired together in a functional manner that permits easy extension [1]. In the classical electronic engineering laboratory, the equipment (bread-boards, pre-fabricated boards and other components) is physically located at a fixed location (inside a laboratory), and the learners have to present in the laboratory concurrently with the equipment in order to use them. Apart from the costs for large learner population, practical classes in electronic engineering are becoming difficult as more and more learners have to use bread-boards and pre-fabricated boards either concurrently (shared) or in a time-limited manner [2].

\section{A. Background}

The education of engineers involves an integral component of hands-on (interactive) work along with delivery of theoretical (sometimes abstract) concepts [3].

This is also true for many science and technology based subjects such as Physics which include a compulsory experimental component [4]. During an actual laboratory experiment, learners enjoy physical contact with the laboratory apparatus (equipment) as well as working together in groups.

The Computer Aided Engineering Education (CAEE) concept shown graphically (shaded box) in Fig. 1 is concerned with the use of computer aids for the enhanced, interactive delivery of educational materials in the field of engineering while maintaining existing pedagogical contents and standards. The CAEE concept consists of two separate components (one for classroom and the other for practical laboratories) related with a similar synergy to that which exists between classroom teaching and practical hands-on laboratory classes.

The CAEE component for hands-on laboratory practical work focuses on the use of video-based augmented reality on mobile devices/platforms with a goal of replicating as much as possible the experience obtainable from an actual physical laboratory through the innovative use of augmented reality technology running on mobile devices.

Augmented reality is still a growing field of research despite the appearance of the technology in the 1960s [5]. 


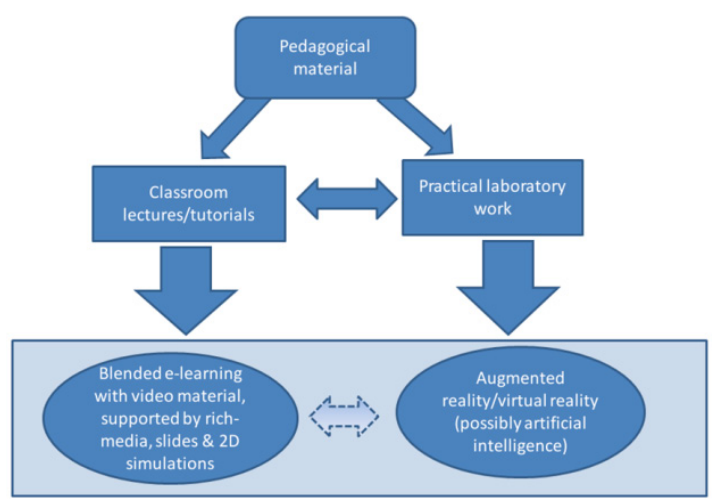

Figure 1. Conceptual overview of Computer Aided Engineering Education (CAEE) scheme

In virtual reality technology, the goal is to immerse a user (viewer) within a virtual environment and so real objects are excluded (or removed) from the environment and replaced by computer generated equivalent models.

Virtual reality systems are usually classified according to the degree of immersion in to three different level of immersion, namely none, semi and fully immersive [6]. Non-immersive virtual reality systems generally do not provide a stereo view of the environment. For example, viewing a virtual reality environment on a typical computer screen is a non-immersive experience as the virtual environment exists only inside the computer screen and interactions with the environment could be through the keyboard, mouse or sometimes joystick devices. Semiimmersive virtual reality systems provide a bigger view of the computer generated environment. This is typically archived through the use of a large screen device or through the use of special eyewear or goggles. In semiimmersive virtual reality systems, special input devices such as wands, special gloves or controllers are also commonly used. The user has a view of both the computer generated environment and the surrounding real world environment. A good example of a semi-immersive virtual reality environment is a gaming station typically used for car racing, in which the output is a combination of one or more large-screen monitors and the input consists of a mock-up driving station complete with steering wheel and foot pedals. Fully-immersive virtual reality systems eliminate completely any reference to the real world environment. This may be archived by wearing special helmet devices with mounted displays or by housing the user in specially designed rooms called CAVEs (Cave Automated Virtual Environments) where all the walls (including floor and ceiling) are replaced by large screens monitors. In both cases, the computer generated environment is projected on the displays or monitors all around the user. Fully-immersive environments also track the user movement particularly orientation and may track the user's gestures and movement for input or optionally use wands, special controllers or special gloves in case tracking of individual finger movements is required for the simulated environment.

Unlike virtual reality, in augmented reality, the goal is not to exclude the real world but to "blend" additional information or computer generated information with realtime information from the real world around a viewer, augmented reality systems are also called mixed reality systems as they attempt to mix both real and virtual objects.
Virtual and augmented reality are often referred to as expensive technologies, especially when special devices and equipment are used [7], however, mobile devices especially smart-phones and tablets devices are a cost effective, technologically accepted solution that may be used as acceptable augmented reality viewers due the improvement in hardware and the wide range of included sensors such as video camera, touch-screen.

\section{DESCRIPTION AND DISCUSSION}

\section{A. Hands-on Laboratory equipment}

The Seeeduino group of pre-fabricated boards (an Arduino compatible board) is sometimes used to teach about embedded sensors in micro-electronics. The stalker line of Seeeduino board shown in Fig. 2 already includes a low-power programmable micro-processor, the AtMega328P and associated circuitry that allows the connection or addition of one or more modular/functional devices such as low-power sensors/wireless radio transmitters alongside basic components such as resistors. The stalker board (Fig. 2) which may be powered from a $+5 \mathrm{v}$ source such as a universal serial bus (USB) port or a battery or from a solar panel device, also includes a real time clock (RTC) circuitary (crystal, chip and CR2032 battery).

At the Telecommunications/ICT for Development (T/ICT4D) Laboratory of the International Centre for Theoretical Phyics (ICTP) located in Trieste, Italy, the Seeeduino stalker v2 board is used in several practical experiments on wireless sensor networking. A fundamental experiment where the intended learning outcome is to introduce the learner to using the board involves the use of the pre-fabricated board in driving a Light Emitting Diode (LED). The experiment requires 2 additional components, an external resistor and light emitting diode in addition to the pre-fabricated board. Power to the board is obtained from a personal computer (or laptop) running the integrated development environment (IDE) supplied by the board manufacturer. The IDE which is used to program the board for this experiment loads the example code (Fig. 3) that may be used to pulse the LED at different frequencies.

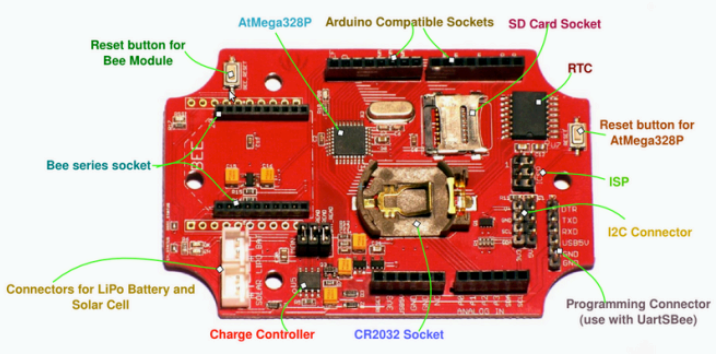

Figure 2. Seeeduino Stalker V2 pre-fabricated board

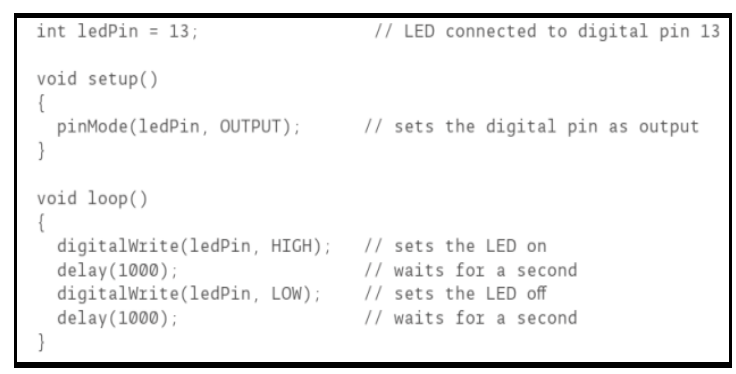

Figure 3. Example software code for pulsating LED [8] 
The intended learning outcome (goal) of the experiment is to familiarize the user with the Seeeduino board is achieved when the learner is able to connect the resistor and LED to the right sockets of the board, power the board via a personal computer running the IDE (with sample program) and obtain the desired results of a pulsating LED.

\section{B. CAEE version}

The CAEE version of the experiment described above uses a low-cost 2D photographic mock up of a Seeduino board and an augmented reality application running on an android tablet to archive/demonstrate the same effect. The android tablet requires a camera which is used to capture and provide the real environment in the mixed reality world. The augmented reality world is composed of computer generated information (text and graphical components) merged with a real-time video feed containing a $2 \mathrm{D}$ photograph (mock-up) of the Seeeduino board.

Fig. 4 shows the startup view of the Seeeduino board's 2D photographic mockup from within the CAEE augmented reality application running on an android tablet device. As shown in Fig. 4, the additional components (resistor and LED) are computer generated objects which only appear when the mock-up is recognized by the application. Also individually components within the board are recognized and highlighted.

Most augmented reality tools work by tracking the outline of an object or (completely abstract) two dimensional marker images such as Quick Response (QR) codes, which may be placed as markers on individual components within the board for tracking [9]. The CAEE implementation thanks to the ability to track outline and contrast levels goes further to also track/identify individual components within the board, without the need to resort to QR codes. This ability allows the use of the CAEE implementation as a smart interactive manual to the Seeeduino board as shown in Fig. 5. That is touching a component brings up a pop-up with textual description of the component, it is possible to provide addition information such as direct clickable links to on-line datasheets and/or other experiments that use the component.

In the procedure, the learner connects the resistor and LED to the board by touching them within the augmented environment. Once both components are attached to the board, the LED begins to pulsate and a new window shows the example code program. Additionally a convenient slider control is added to allow the learner to quickly vary the LED pulsation duration.

Fig. 6 shows the completed running experiment with the pulsating LED.

An additional procedure for disconnecting and reconnecting the components from the board is included in the view of the final running experiment; that is when the LED or resistor is touch would disconnect one pin of the components from the board; subsequently touching the component again will re-attach it to the board. This is used to teach the learners the importance of disconnecting components before making changes.

\section{Discussion}

The CAEE implementation is quite real or lifelike when compared to the original experiment additionally the same functionality (pulsating LED) is obtained from the CAEE implementation. The $2 \mathrm{D}$ photographic mock-up can be

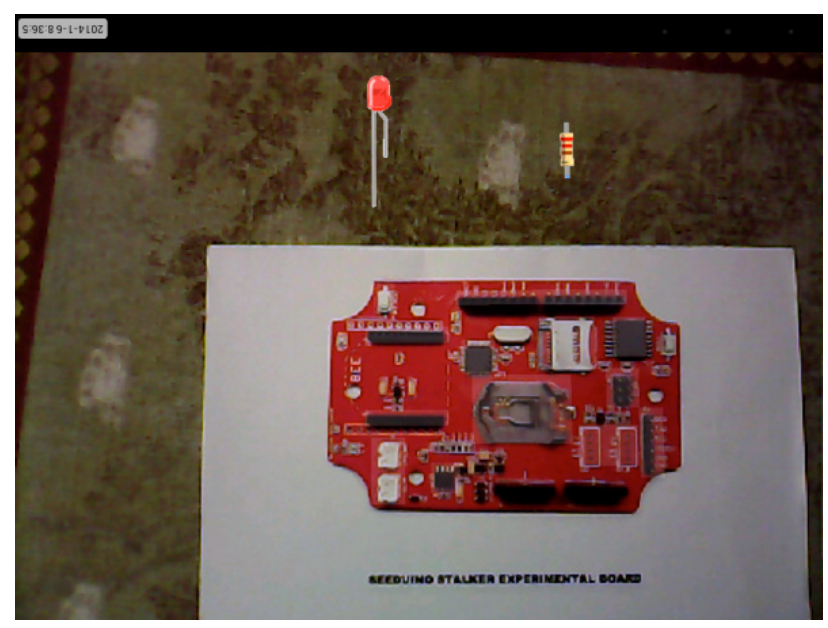

Figure 4. Initial view of CAEE implementation

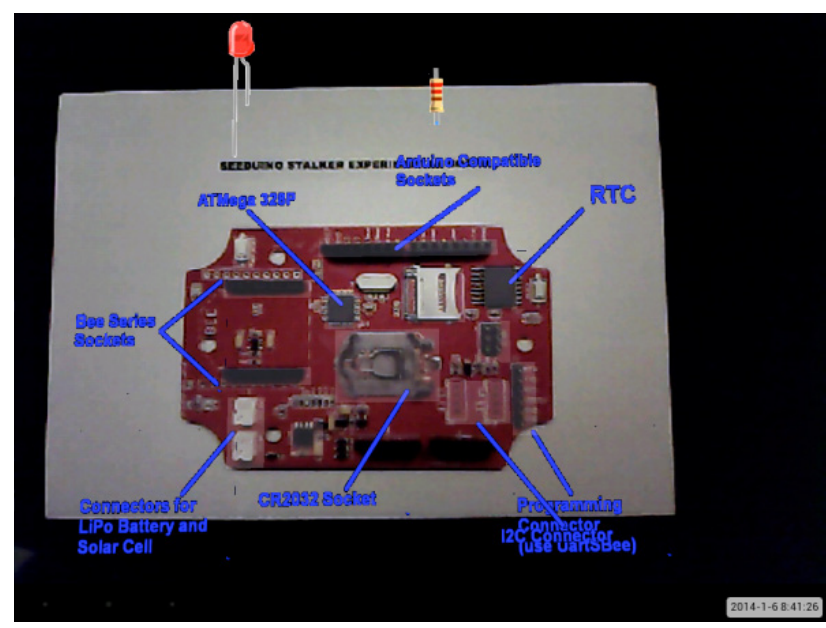

Figure 5. CAEE implementation as a smart interactive manual

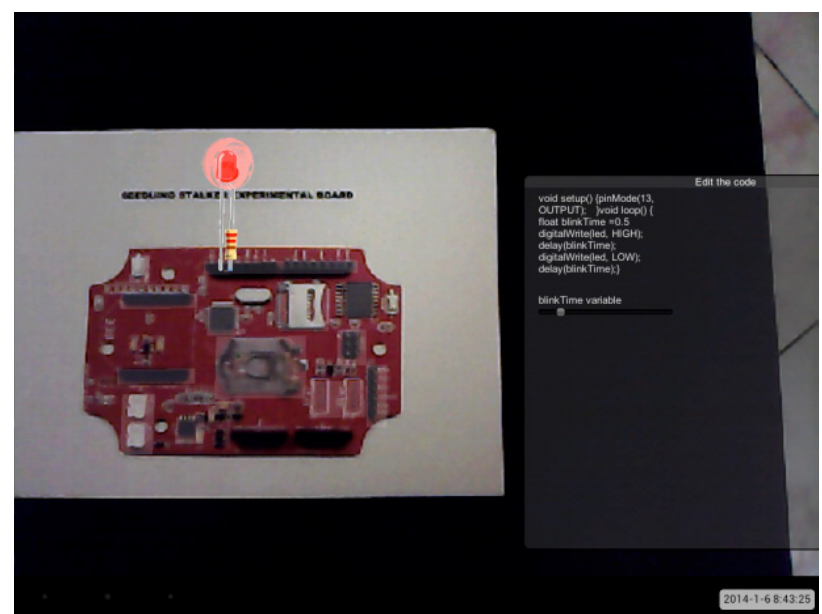

Figure 6. View of completed running experiment in CAEE implementation

printed using a low cost color printer at scales of $1: 1$ or even lower. The same instructional material is used for both versions of the experiment, in the CAEE implementation connection of components is archived by touching; this represents a minimal change in instructional material.

During practical testing, it was quickly noted that the CAEE implementation also tracks successfully the real Seeeduino board (not just its photographic mock-up) even from different angles of view, distances and resolution. As 
this implies, the CAEE component may be used as an aid during practical laboratory sessions (on the real board), in addition to group collaborative work involving multiple learners standing around either the photographic mock-up or the real board. In the CAEE implementation, although it is possible to implement a drag-and-drop motion for attaching the components, a simple touch action is used in a bid to focus on showing the correct connection of components.

Mobile augmented reality is increasingly being used mainly for visualization especially in the medical fields. The CAEE concept is one of the first (if not the first) use of this technology for experiments. A similar independent work is documented in the "Augmented Reality Breadboarding" [10]. Unlike in most virtual reality implementations, the CAEE concept is about simulating the experimental procedures/outcomes and not the simulation of devices/components. Unlike remote laboratories, a central experimental facility is not involved and the CAEE implementation may be used on mobile devices already owned by the learners, even in the comfort of their homes.

\section{CONCLUSION}

This paper highlights some of innovative features of the CAEE version of an experiment such as the ability to closely implement the step-by-step procedure of existing laboratory based hands-on experiments; lower costs (especially if learners use their own mobile device) and the ability use off-line (on-line aspects would be mainly used for submissions of analytics such as number of attempts \& completion time-stamp). Importantly, it is noted that the Seeeduino board functionality is not simulated but rather the expected outcome of inter-connecting both the LED and resistor to the Seeeduino board is simulated.

\section{ACKNOWLEDGMENT}

The augmented reality component and android application is based on the prior work and contributions from Engineer. M. Santachiara of Santa's Co, Reggio Emilia, Italy.

\section{REFERENCES}

[1] C. Onime, J. Uhomoibhi, and M. Zennaro, "Demonstration of a low cost implementation of an existing hands-on laboratory experiment in electronic engineering," in REV2014: 11th International Conference on Remote Engineering and Virtual Instrumentation (REV), Porto, 2014.

[2] S. A. Shanab, S. Odeh, R. Hodrob, and M. Anabtawi, "Augmented Reality Internet Labs Versus Hands-On and Virtual Labs: A
Comparativ Study," in International Conference on Mobile and Computer Aided Learning (IMCL), Amman, 2012, pp. 17-21. http://dx.doi.org/10.1109/IMCL.2012.6396444

[3] Chad E. Davis, Mark B. Yeary, and James J. Sluss, "Reversing the Trend of Engineering Enrollment Declines With Innovative Outreach, Recruiting and Retention programs," IEEE TRANSACTIONS ON EDUCATION, vol. 55, no. 2, pp. 157-163, 2012. http://dx.doi.org/10.1109/TE.2011.2157921

[4] Galiya Sharafutnova et al., "Distance Learning laboratory: a remote Atomic and Nuclear Physics experiment," Australian Physics, vol. 50, no. 2, pp. 55-59, April 2013.

[5] P. Milgram and F. Kishino, "A Taxonomy of Mixed Reality Visual Displays," IEICE Transactions on Information Systems, vol. E77-D, no. 12, pp. 1321-1329, 1994.

[6] J. Fox, B. Arena, and J. N Bailenson, "Virtual Reality: A Survival Guide for the Social Scientist," Journal of Media Psychology, vol. 21, no. 3, pp. 95-113, 2009. http://dx.doi.org/10.1027/18641105.21.3.95

[7] Elizabeth FitzGerald et al., "Augmented reality and mobile learning: the state of the art," in 11th World Conference on Mobile and Contextual Learning (mLearn 2012), Helsinki, 2012, pp. 6269.

[8] Arduino. (2013, May) Arduino - DigitalWrite. [Online]. http://arduino.cc/en/Reference/digitalWrite

[9] J. M. Andujar, A. Mejias, and M. A. Marquez, "Augmented Reality for the Improvement of Remote Laboratories: An Augmented Remote Laboratory," IEEE TRANSACTIONS ON EDUCATION, vol. 54, no. 3, pp. 492-500, 2011. http://dx.doi.org/10.1109/TE.2010.2085047

[10] Adam Fabio. (2013, November) Augmented Reality Breadboarding. [Online]. http://hackaday.com/2013/11/06/aug mented-reality-breadboarding/

\section{AUTHORS}

C. Onime is with the Abdus Salam International Centre for Theoretical Physics, Strada Costiera 11, Trieste, 34100, Italy (e-mail: clement.onime@ictp.it).

M. Zennaro is with Abdus Salam International Centre for Theoretical Physics, Strada Costiera 11, Trieste, 34100, Italy (e-mail: mzennaro@ictp.it).

J. Uhomoibhi is with the University of Ulster, Antrim Road, Newtownabbey, Belfast, Northern Ireland (e-mail: j.uhomoibhi@ulster.ac.uk).

This work was jointly supported by the Telecommunications/ICT for Development (T/ICT4D) Laboratory and the Information, Communication and Telecommunications Section (ICTS), both of the Abdus Salam International Centre for Theoretical Physics (ICTP). It is an extended and modified version of a paper presented at the International Conference on Remote Engineering \& Virtual Instrumentation (REV2014), held in Porto, Portugal, 26 - 28 February 2014. Submitted 26 March 2014. Published as resubmitted by the authors 05 October 2014. 